\title{
Making light work with triboelectric energy conversion
}

\author{
Chenguo $\mathrm{Hu}^{1, *}$ (D) \\ ${ }^{1}$ Department of Applied Physics, Chongqing University, Chongqing 400044, People's Republic of China
}

Received: 20 February 2019
Accepted: 20 February 2019
Published online:
11 March 2019
C Springer Science+Business
Media, LLC, part of Springer
Nature 2019

As the world is entering the era of internet of things (IOT) and artificial intelligence, microelectronic devices have been developing rapidly towards the trend of more miniaturization, mobility and multifunctionality. Traditional batteries such as the nickel metal hydride battery and lithium battery are no longer suitable to drive the worldwide sensor network that will emerge in the near future. Therefore, to ensure the success of the IOT it is very urgent to develop a widely distributed mobile power, which can come from solar energy, thermal energy, wind energy and mechanical triggers or vibrations. Even though it is not enough to provide consistent power to the main grid, it is very suitable for the mobile sensor and self-powered system.

In 2012, Z. L. Wang's team at Georgia Tech invented a new type of mechanical energy harvester, namely the triboelectric nanogenerator (TENG) [1]. TENGs use the ubiquitous triboelectrification-induced polarization changes to surface charge as materials' contact separation varies under periodic applied force, thus producing a displacement current that drives electrons to flow between electrodes. In such a working process, the mechanical energy existing in the environment or organisms can be converted into electricity. Currently, research on nanogenerators has inspired worldwide interest because of their importance not only as a power source, but also self-powered sensors with applications ranging from IOT, environmental monitoring, health care, medical science, infrastructure monitoring and security. Therefore, TENG has become one of the best choices for a new era of energy.

The surface charge density on friction materials determines the output voltage, current and power of a TENG, so a lot of effort has been devoted to improving the friction charge density, such as the modifying material composition, increasing effective contact area and controlling environmental conditions.

Featuring on the cover of this issue (Fig. 1), Q. L. Song's research team from Southwest University in China presents an ingenious photoelectric hybrid TENG based on organolead halide $\mathrm{MAPbI}_{x} \mathrm{Cl}_{3-\mathrm{x}}$

Address correspondence to E-mail: hucg@cqu.edu.cn 
Figure 1 Schematic of the photovoltaic triboelectric nanogenerator featured in [2].

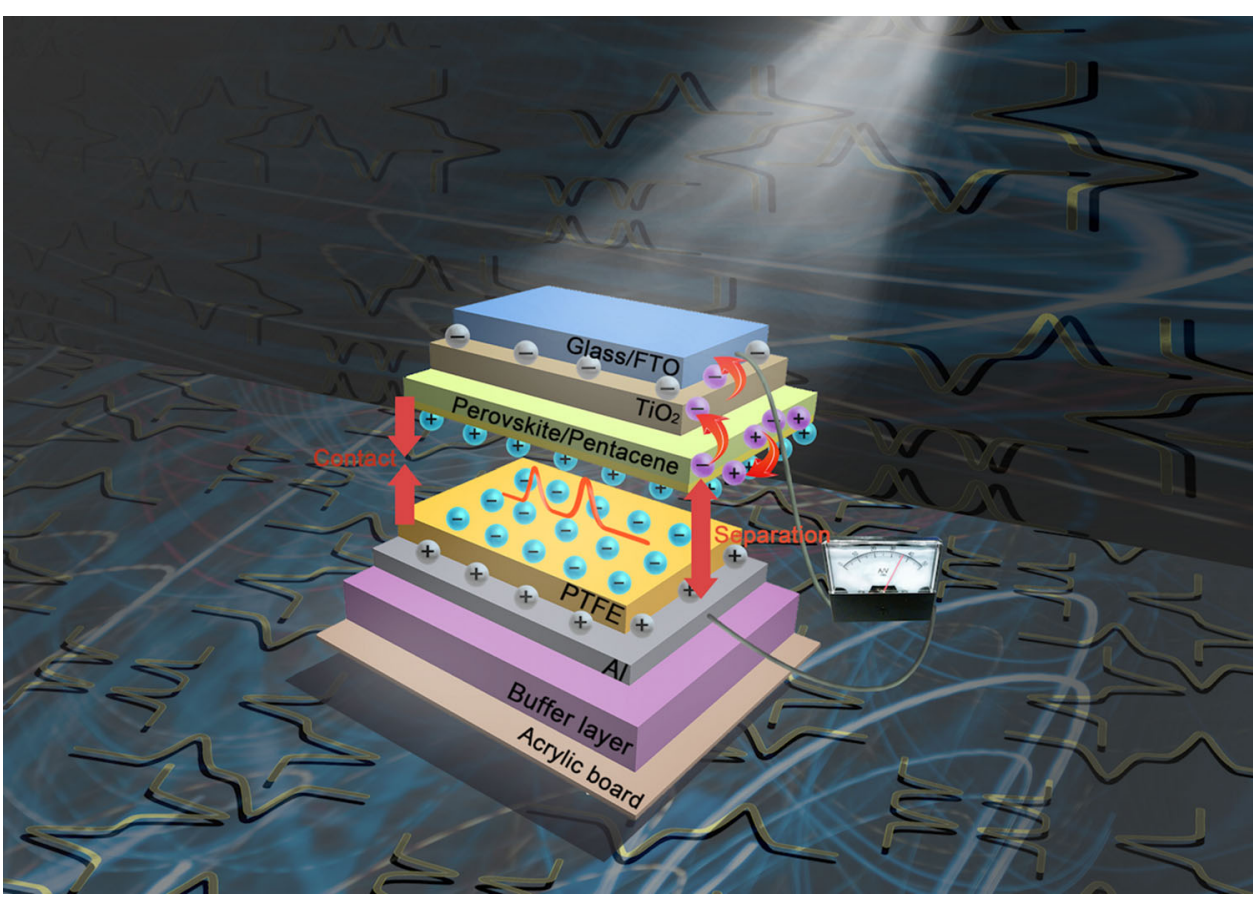

perovskite as its functional material [2]. These perovskites have been more commonly applied in solar cells in the past several years. In their device, the perovskite is not only the friction layer, but also the light absorber. Based on the coupling of photoelectric-triboelectric conversion, vibrational mechanical energy and solar energy are collected at the same time and converted into electricity, improving the output performance of the proposed TENG. The device structure includes for the first time both a $\mathrm{TiO}_{2}$ electron-transport layer and a pentacene hole-transport layer. This combination aligns the energy levels for effective charge transfer. The photo-induced performance was additionally enhanced by the close interaction between the two charge transport layers.

This study has achieved multiple energy collection modes in a single TENG device, which provides a new approach and methodology to further improve the TENG output and is expected to exhibit potential applications in photodetection.

\section{References}

[1] Fan FR, Tian ZQ, Wang ZL (2012) Flexible triboelectric generator. Nano Energy 1:328-334. https://doi.org/10.1016/j. nanoen.2012.01.004

[2] Yang XD, Han JJ, Wang G, Liao LP, Xu CY, Hu W, Li P, Wu B, Elseman AM, Zhou GD, Song QL (2019) Robust perovskite-based triboelectric nanogenerator enhanced by broadband light and interface engineering. J Mater Sci 54:1-13. https://doi.org/10.1007/s10853-019-03351-9

Publisher's Note Springer Nature remains neutral with regard to jurisdictional claims in published maps and institutional affiliations. 